\title{
Dynamical and point symmetry of the Kondo effect in triangular quantum dot
}

\author{
${ }^{1}$ T. Kuzmenko, ${ }^{1}$ K. Kikoin, and ${ }^{1,2}$ Y. Avishai \\ ${ }^{1}$ Department of Physics, Ben-Gurion University of the Negev, \\ Beer-Shea 84105, Israel \\ 2 Ilse Katz Center for Nano-Technology, \\ Ben-Gurion University of the Negev, \\ Beer-Sheva 84105, Israel
}

(Dated: December 22, 2018)

\begin{abstract}
In this work we concentrate on the point symmetry of triangular triple quantum dot and its interplay with the spin rotation symmetry in the context of Kondo tunneling through this kind of artificial molecule. A fully symmetric triangular triple quantum dot is considered, consisting of three identical puddles with the same individual properties (energy levels and Coulomb blockade parameters) and inter-dot coupling (tunnel amplitudes and electrostatic interaction). The underlying Kondo physics is determined by the product of a discrete rotation symmetry group in real space and a continuous rotation symmetry in spin space. These symmetries are reflected in the resulting exchange hamiltonian which naturally involves spin and orbital degrees of freedom. The ensuing poor-man scaling equations are solved and the Kondo temperature is calculated.
\end{abstract}

\section{INTRODUCTION}

The analogy between complex quantum dots and real molecules was recognized both by experimentalists and theoreticians at early stage of studies of these artificial nanoobjects (see, e.g. 1.2 ). Soon after the discovery of the Kondo effect in tunneling through quantum dots $(\mathrm{QD})^{3}$, it was recognized that this phenomenon may be realized in tunneling through complex quantum dots consisting of two or three electron puddles (double ant triple quantum


configurations, where the dots are ordered linearly either parallel or perpendicular to the metallic leads. Meanwhile, modern experimental methods allow also fabrication of quantum dots in a triangular geometry. Triangular triple quantum dot (TTQD) was considered theoretically ${ }^{\frac{8}{}}$ and realized experimentally very recently,$\underline{9}$ in order to demonstrate the ratchet effect in single electron tunneling. To achieve this effect the authors proposed a configuration, where two of the three puddles are coupled in series with the leads (source and drain), while the third one has a tunnel contact with one of its counterparts and only a capacitive coupling with the other.

In this paper we concentrate on the point symmetry of TTQD and its interplay with the spin rotation symmetry in a context of Kondo tunneling through this artificial molecule. Indeed, the generic feature of Kondo effect is the involvement of internal degrees of freedom of localized "scatterer" in the interaction with continuum of electron-hole pair excitations in the Fermi sea of conduction electrons. These are spin degrees of freedom in conventional Kondo effect, although in some cases the role of pseudospin may be played by configuration quantum numbers, like in

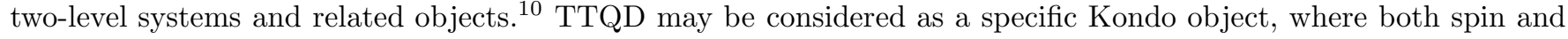
configuration excitations are involved in cotunneling on equal footing.

To demonstrate this interplay, we consider a fully symmetric TTQD consisting of three identical puddles with the same individual properties (energy levels and Coulomb blockade parameters) and inter-dot coupling (tunnel amplitudes and electrostatic interaction. Like in the above mentioned triangular ratchet ${ }^{8.9}$, we assume that the TTQD in the ground state is occupied by one electron and Coulomb blockade is strong enough to completely suppress double occupancy of any valley $j=1,2,3$. This means that the only mechanism of electron transfer through TTQD is cotunneling, where one electron leaves the valley $j$ for metallic leads, whereas another electron tunnels from reservoir to the same valley $j$ or to another valley $l$. In the former case only the spin reversal is possible, whereas in the latter case not only the spin is affected but also the TTQD is effectively "rotated" either clockwise or anti-clockwise (see Fig. 1).

Discrete rotation in real space and continuous rotation in spin space may be described in terms of group theory. The group $C_{3 v}$ characterizes the symmetry of triangle, and the group $S U(2)$ describes the symmetry of spin $1 / 2$. So the total symmetry of TTQD is determined by direct product of these two groups. One may use an equivalent language of permutation group $P_{3}$ for description of configuration of TTQD with an electron occupying one of three possible positions in its wells. 




FIG. 1: Triangular triple quantum dot (TTQD) in a contact with source (s) and drain (d) leads. Clockwise (c) and anti-clockwise (a) "rotation" of TTQD due to cotunneling through the channels $V_{s 3}$ and $V_{s 2}$, respectively.

\section{HAMILTONIAN OF ARTIFICIAL TRIANGULAR MOLECULE}

A symmetric TTQD in a contact with source and drain leads (Fig. 1) is described by the Hamiltonian

$$
H=H_{d}+H_{l e a d}+H_{t}
$$

The first term $H_{d}$ is the Hamiltonian of the isolated TQD,

$$
H_{d}=\epsilon \sum_{j=1}^{3} \sum_{\sigma} d_{j \sigma}^{\dagger} d_{j \sigma}+Q \sum_{j} n_{j \uparrow} n_{j \downarrow}+Q^{\prime} \sum_{\langle j l\rangle} \sum_{\sigma} n_{j \sigma} n_{l \sigma^{\prime}}+W \sum_{\langle j l\rangle} \sum_{\sigma}\left(d_{j \sigma}^{\dagger} d_{l \sigma}+H . c .\right)
$$

where $\sigma=\uparrow, \downarrow$ is the spin index, $\langle j l\rangle=\langle 12\rangle,\langle 23\rangle,\langle 31\rangle . Q$ and $Q^{\prime}$ are intra-dot and inter-dot Coulomb blockade parameters, $W_{j l}$ are inter-dot tunneling parameters. The second term $H_{l e a d}$ describes the electrons in the source $(s)$ and drain $(d)$ electrodes,

$$
H_{l e a d}=\sum_{k \sigma} \sum_{b=s, d} \epsilon_{k b} c_{k b \sigma}^{\dagger} c_{k b \sigma}
$$

The last term $H_{t}$ is the tunneling Hamiltonian

$$
H_{t}=\sum_{k \sigma}\left(\sum_{j=2,3} V_{s j} c_{k s j \sigma}^{\dagger} d_{j \sigma}+V_{s} d_{k d \sigma}^{\dagger} d_{1 \sigma}+H . c .\right)
$$

which describes a configuration with two-channel tunneling between the TTQD and the source and single-channel tunneling between the TTQD and the drain. If all three channels are equivalent,

$$
V_{d}=V_{s 2}=V_{s 3} \equiv V
$$


the tunnel contacts preserve the rotation/permutation symmetry of TTQD. If all tunnel constants are different, this symmetry is completely destroyed by tunneling. In the intermediate case

$$
V_{d} \neq V_{s 2}=V_{s 3} \equiv V_{s}
$$

the system preserves one mirror reflection axis $(2 \leftrightarrow 3)$. The Kondo tunneling regime will be analyzed both in cases (5) and (6).

The Hamiltonian $H$ (1) is easily diagonalized by using the following set of basis functions $|j \sigma\rangle=d_{j \sigma}^{\dagger}|0\rangle(j=1,2,3)$. The eigenfunctions of symmetric TTQD in this basis are

$$
\begin{aligned}
\Psi_{\sigma, A_{1}} & =\frac{1}{\sqrt{3}}(|1 \sigma\rangle+|2 \sigma\rangle+|3 \sigma\rangle), \\
\Psi_{\sigma, E(+)} & =\frac{1}{\sqrt{3}}\left(|1 \sigma\rangle+e^{2 i \varphi}|2 \sigma\rangle+e^{i \varphi}|3 \sigma\rangle\right), \\
\Psi_{\sigma, E(-)} & =\frac{1}{\sqrt{3}}\left(|1 \sigma\rangle+e^{i \varphi}|2 \sigma\rangle+e^{2 i \varphi}|3 \sigma\rangle\right)
\end{aligned}
$$

with $\varphi=2 \pi / 3$. Here $A_{1}$ and $E$ are two irreducible representations of the group $C_{3 v}$ The spin states with $\mathcal{N}=1$ are spin doublets $(D)$, so the Hamiltonian of isolated TTQD in this charge sector has six eigenstates $|D A\rangle,|D E\rangle$. The single electron energies are

$$
E_{D A_{1}}=\epsilon+2 W, \quad E_{D E}=\epsilon-W \text {. }
$$

First of these energy levels is a conventional spin doublet with fully symmetrical "orbital" wave function $\Psi_{\sigma, A_{1}}$. The second one is doubly degenerate both in spin and orbital quantum number.

A peculiar feature of the three-site configuration is an explicit dependence of the order of levels in spin multiplet on the sign of tunnel integral $W$. The ground state is a spin doublet $E_{D A_{1}}$, provided $W<0$. In case of $W>0$ the lowest level is the orbital doublet $E_{D E}$. The orbital degeneracy of the states $E( \pm)$ is a manifestation of rotation/permutation degrees of freedom of TTQD illustrated by Fig. 1. These discrete rotations are explicitly involved in Kondo tunneling.

\section{KONDO TUNNELING THROUGH PARTIALLY OCCUPIED TTQD}

A contact between symmetric TTQD and leads in the two-terminal geometry preserves rotation symmetry $C_{3 v}\left(P_{3}\right)$ unless the tunnel Hamiltonian $H_{t}$ has lower symmetry than TTQD. In any case it is useful to re-expand the tunnel Hamiltonian in partial waves, which respect the "point symmetry" of the Hamiltonian $H$. Such ansatz is known in the theory of Kondo effect in 3D metal, where the spherical partial wave representation for Bloch electrons in conduction band was introduced in Ref. 11]. Later on, this approach was extended by many authors to the case of point crystal group representation (see, e.g., the review [10]). Here we also meet the situation where the triangular quantum dot imposes its point symmetry on the continuum of electron states in the leads under certain conditions.

If the condition (5) is satisfied, the point symmetry of the device as a whole is still $C_{3 v}$, and the band states may be re-expanded as

$$
\begin{aligned}
c_{A, \mathbf{k}, \sigma} & =\frac{1}{\sqrt{3}}\left(c_{d, \mathbf{k}, \sigma}+c_{s_{2}, \mathbf{k}, \sigma}+c_{s_{3}, \mathbf{k}, \sigma}\right) . \\
c_{E(+), \mathbf{k}, \sigma} & =\frac{1}{\sqrt{3}}\left(c_{d, \mathbf{k}, \sigma}+e^{2 i \varphi} c_{s_{2}, \mathbf{k}, \sigma}+e^{i \varphi} c_{s_{3}, \mathbf{k}, \sigma}\right), \\
c_{E(-), \mathbf{k}, \sigma} & =\frac{1}{\sqrt{3}}\left(c_{d, \mathbf{k}, \sigma}+e^{i \varphi} c_{s_{2}, \mathbf{k}, \sigma}+e^{2 i \varphi} c_{s_{3}, \mathbf{k}, \sigma}\right),
\end{aligned}
$$

which has the same angular dependence as (17).

The Anderson Hamiltonian $H$ rewritten in these variables may be expressed by means of Hubbard operators $X^{\lambda \lambda^{\prime}}=|\lambda\rangle\left\langle\lambda^{\prime}\right|$, with $\lambda=0, \gamma, \Gamma, \Lambda$ :

$$
\begin{aligned}
H & =E_{0} X^{00}+\sum_{\lambda} E_{\lambda} X^{\lambda \lambda}+\sum_{\Gamma} E_{\Gamma} X^{\Gamma \Gamma}+\sum_{k \sigma} \sum_{k \gamma} \varepsilon_{k} n_{k \gamma} \\
& +\sum_{\gamma}\left[V^{\gamma 0} X^{\gamma 0} c_{\gamma}+\sum_{\Gamma \gamma \gamma^{\prime}} V^{\gamma \Gamma} c_{\gamma}^{\dagger} X^{\gamma \Gamma}+\text { h.c. }\right]
\end{aligned}
$$


Here $|0\rangle$ stands for an empty TTQD, $|\gamma\rangle=\left|D A_{1}\right\rangle,|D E\rangle$ belong to the single electron charge sector, and $|\Gamma\rangle$ are the eigenvectors of two-electron states. The eigenstates $E_{\Gamma}$ for $\mathcal{N}=2$ are

$$
\begin{aligned}
E_{S A_{1}} & =\epsilon_{2}+2 W-\frac{8 W^{2}}{Q}, \\
E_{T E} & =\epsilon_{2}+W, \\
E_{S E} & =\epsilon_{2}-W-\frac{2 W^{2}}{Q}, \\
E_{T A_{2}} & =\epsilon_{2}-2 W .
\end{aligned}
$$

Here $\epsilon_{2}=2 \epsilon+Q^{\prime}$, indices $S, T$ denote spin singlet and spin triplet configurations of two electrons in TTQD, and the inequality $W \ll Q$ is used explicitly. The irreducible representation $A_{2}$ contains two-electron eigenfunction, which is odd with respect to permutations $j \leftrightarrow l$. Like in the singly occupied TTQD, the level ordering is sensitive to the sign of tunnel integral $W$.

The tunnel matrix elements are redefined accordingly.

$$
V^{0 \gamma}=\left\langle\psi_{c, \gamma}\left|H_{t}\right| \Psi_{\gamma}\right\rangle, \quad V^{\gamma \Gamma}=\left\langle\psi_{c, \gamma^{\prime}}, \Psi_{\gamma}\left|H_{t}\right| \Psi_{\Gamma}\right\rangle .
$$

The matrix elements intermixing the charge sectors $\mathcal{N}=1,2$, add conduction electron $\gamma^{\prime}$ to the dot electron $\gamma$, and the resulting two-electron states $\Gamma$ arise in accordance with Clebsch-Gordan coefficients both in spin and orbital subspaces $\mathcal{C}_{\rho \rho^{\prime}}^{\rho^{\prime \prime}} \mathcal{C}_{\sigma \sigma^{\prime}}^{M}$ where $\rho=A, E( \pm), M=S, T$.

We describe Kondo tunneling by means of Haldane-Anderson renormalization group (RG) approach ${ }^{12.13}$. According to this procedure the parameters of the original Hamiltonian are renormalized in the course of rescaling of the energy $\mathcal{D}$ characterizing the width of conduction electron continuum in the leads from original value $\mathcal{D}_{0}$ to the energy $T_{K}$, which characterizes Kondo correlations. The latter is found from the solution of scaling equations. First, the energy levels $E_{\gamma}$ are renormalized by the virtual excitations of the states $|0\rangle$ and $|\Gamma\rangle$. The flow equations have the form

$$
\begin{aligned}
& E_{A}(\mathcal{D})=\epsilon+2 W-\Delta \ln \left(\mathcal{D}_{0} / \mathcal{D}\right), \\
& E_{E}(\mathcal{D})=\epsilon-W-\Delta \ln \left(\mathcal{D}_{0} / \mathcal{D}\right),
\end{aligned}
$$

where $\Delta \sim \rho_{0} V^{2}$ is the tunneling rate, $\rho_{0}$ is the density of electron states in the leads. This scaling ends at $\mathcal{D} \rightarrow \overline{\mathcal{D}}$, with $\overline{\mathcal{D}}$ defined as

$$
E_{\gamma}(\overline{\mathcal{D}}) \sim \overline{\mathcal{D}}
$$

(Schrieffer-Wolff limit) $\frac{12}{2}$. Besides, the Haldane-Anderson procedure generates effective two-particle vertices $J_{\gamma}$, which describe effective exchange interaction between dot and lead electrons. Various cotunneling processes contribute to this interaction. In conventional Kondo problem the effective low-energy exchange Hamiltonian has the form $J \mathbf{S} \cdot \mathbf{s}$, where $\mathbf{S}$ and $\mathbf{s}$ are the spin operators for dot and lead electrons, respectively $\stackrel{13}{\underline{13}}$. Here we are in a position, where the low-energy states of the quantum dot are represented by a multiplet containing both spin and orbital indices. In this case the form of effective Hamiltonian is predetermined by a dynamical symmetry of the Hamiltonian $H_{a}{ }^{5.14}$.

The dynamical symmetry group of a given Hamiltonian is determined not only by the operators, which leave this Hamiltonian invariant but also by the operators describing transitions between different energy levels of the multiplet. It follows from this definition that the dynamical symmetry may vary depending on the set of energy levels which fall into an actual energy interval. In particular, it changes in the process of rescaling the energy interval in the Anderson's RG procedure $\frac{13}{2}$. If the condition $\overline{\mathcal{D}} \sim 3 W$ is satisfied, then the dynamical symmetry is determined by the whole multiplet $E_{\gamma}$. The dynamical symmetry group, which describes all possible transitions within the set $\left\{D A_{1}, D E( \pm)\right\}$ is $S U(6)$. Among 35 generators of this group are nine spin vectors $\mathbf{S}_{\rho \rho^{\prime}}$ with projections defined as

$$
\begin{array}{r}
S_{\rho \rho^{\prime}}^{+}=X^{\uparrow \rho, \downarrow \rho^{\prime}}, \quad S_{\rho \rho^{\prime}}^{-}=X^{\downarrow \rho, \uparrow \rho^{\prime}}, \\
S_{\rho \rho^{\prime}}^{z}=\frac{1}{2}\left(X^{\uparrow \rho, \uparrow \rho^{\prime}}-X^{\downarrow \rho, \downarrow \rho^{\prime}}\right) .
\end{array}
$$

The rest 8 operators describe the permutation degrees of freedom of TTQD. In the process of further reduction of the energy scale $\mathcal{D}$ the highest of two levels $E_{\gamma}$ is quenched, and eventual symmetry of Kondo effect depends on the sign of tunneling amplitude $W$.

In case of $W<0$ the permutation degrees of freedom are quenched at low-energy scale. The only vector, which is involved in the Kondo cotunneling through TTQD is the spin $\mathbf{S}_{A A}$ defined in Eq. (13). The lead states are still classified in accordance with the point symmetry of the system. As a result the SW Hamiltonian has the form

$$
H_{S W}=J_{E}\left(\mathbf{S} \cdot \mathbf{s}_{+}+\mathbf{S} \cdot \mathbf{s}_{-}\right)+J_{A} \mathbf{S} \cdot \mathbf{s}_{A},
$$


(the subindex $A$ of spin operator of TQD is omitted). The exchange vertices $J_{\rho}$ are

$$
\begin{aligned}
& J_{E}=-\frac{2 V^{2}}{3}\left(\frac{1}{\epsilon+Q^{\prime}-\epsilon_{F}}-\frac{1}{\epsilon+Q-\epsilon_{F}}\right) \\
& J_{A}=\frac{2 V^{2}}{3}\left(\frac{3}{\epsilon_{F}-\epsilon}+\frac{1}{\epsilon+Q-\epsilon_{F}}+\frac{2}{\epsilon+Q^{\prime}-\epsilon_{F}}\right) .
\end{aligned}
$$

The constant $J_{A}$ has antiferromagnetic sign like in conventional SW case, whereas the constant $J_{E}$ is negative due to the inequality $Q \gg Q^{\prime}$.

Formally, the Hamiltonian (14) describes three-channel cotunneling 15 However, two of three available exchange channels in the Hamiltonian (14) are irrelevant for Kondo cotunneling, because the coupling constant $J_{E}$ is negative (ferromagnetic). As a result the conventional Kondo regime arises in $D A$ channel with Kondo temperature

$$
T_{K}^{(A)}=\bar{D} \exp \left\{-\frac{1}{\rho_{0} J_{A}}\right\} .
$$

where $\rho_{0}$ is the density of electron states in the leads, which is assumed to be the same for all channels. In case of $W>0$ the doublet $E_{D A}$ is quenched at $\bar{D}<W$, and the Kondo effect is defined by the tunneling through TTQD in the state $|D, E\rangle$ whose symmetry is $S U(4)$. Similar situation was observed for a double quantum dot in series geometry ${ }^{16}$ In that case the pseudospin variable describes two possible occupations of an electron in a double dot. The 15 generators of this group bunch in four spin vectors $\mathbf{S}_{\rho \rho^{\prime}}$ with $\rho=E( \pm)$ ) and one pseudospin vector $\mathcal{T}$ defined as

$$
\begin{aligned}
& \mathcal{T}^{+}=\sum_{\sigma} X^{\sigma+, \sigma-}, \quad \mathcal{T}^{-}=\sum_{\sigma} X^{\sigma-, \sigma+} \\
& \mathcal{T}^{z}=\frac{1}{2} \sum_{\sigma}\left(X^{\sigma+, \sigma+}-X^{\sigma-, \sigma-}\right) .
\end{aligned}
$$

Here the indices \pm stand for "orbital" indices $E( \pm)$ for the sake of brevity.

Due to high degeneracy of the ground state of TTQD, the effective SW Hamiltonian acquires quite complicated form,

$$
\begin{aligned}
H_{S W} & =J_{1}\left(\mathbf{S}_{+} \cdot \mathbf{s}_{+}+\mathbf{S}_{-} \cdot \mathbf{s}_{-}\right)+J_{2}\left(\mathbf{S}_{+} \cdot \mathbf{s}_{-}+\mathbf{S}_{-} \cdot \mathbf{s}_{+}\right) \\
& +J_{3}\left(\mathbf{S}_{+}+\mathbf{S}_{-}\right) \cdot \mathbf{s}_{A}+J_{4}\left(\mathbf{S}_{+-} \cdot \mathbf{s}_{-+}+\mathbf{S}_{-+} \cdot \mathbf{s}_{+-}\right) \\
& +J_{5}\left(\mathbf{S}_{+-} \cdot\left(\mathbf{s}_{A-}+\mathbf{s}_{+A}\right)+\mathbf{S}_{-+} \cdot\left(\mathbf{s}_{A+}+\mathbf{s}_{-A}\right)\right) \\
& +J_{6} \mathcal{T} \cdot \boldsymbol{\tau}
\end{aligned}
$$

where

$$
\begin{aligned}
\tau_{z} & =\frac{1}{2} \sum_{\sigma}\left(c_{\sigma_{+}}^{\dagger} c_{\sigma_{+}}-c_{\sigma_{-}}^{\dagger} c_{\sigma_{-}}\right) \\
\tau^{+} & =\sum_{\sigma} c_{\sigma_{+}}^{\dagger} c_{\sigma_{-}}, \quad \tau^{-}=\left(\tau^{+}\right)^{\dagger}
\end{aligned}
$$

and the coupling constants $J_{1-6}$ are

$$
\begin{aligned}
J_{1} & =J_{4}=\frac{2 V^{2}}{3}\left(\frac{3}{\epsilon_{F}-\epsilon}\right. \\
& \left.+\frac{1}{\epsilon+Q-\epsilon_{F}}+\frac{2}{\epsilon+Q^{\prime}-\epsilon_{F}}\right) . \\
J_{2} & =J_{3}=J_{5} \\
& =-\frac{2 V^{2}}{3}\left(\frac{1}{\epsilon+Q^{\prime}-\epsilon_{F}}-\frac{1}{\epsilon+Q-\epsilon_{F}}\right) . \\
J_{6} & =\frac{V^{2}}{\epsilon_{F}-\epsilon}+\frac{V^{2}}{\epsilon+Q^{\prime}-\epsilon_{F}} .
\end{aligned}
$$

Both spin and "orbital" degrees of freedom of TTQD are involved in effective Kondo tunneling. The indirect exchange coupling constants arise as combinations of cotunneling processes with virtual excitation of states with zero 
and two electrons. It is worth mentioning that the rotation $C_{3 v}$ symmetry in a system dot+leads may be violated in the process of cotunneling unlike the rotation spin symmetry [see the terms $\sim J_{3,5}$ in the Hamiltonian (18)].

Some of these constants are positive $\left(J_{1,4,6}\right)$ like in conventional SW transformation, and some are negative $\left(J_{2,3,5}\right)$. Thus the Kondo effect arises as a result of interplay of antiferromagnetic and ferromagnetic exchange involving both spin and pseudospin variables. This interplay is described by scaling equations obtained in a framework of Anderson's "poor man scaling" procedure $e^{13}$. The system of scaling equations describes the flow of exchange vertices, with reducing the energy scale $\bar{D}$ from the boundary value $\bar{D}_{S W}$ to the Kondo temperature $T_{K}$. It has the following form:

$$
\begin{aligned}
& \frac{d j_{1}}{d t}=-\left[j_{1}^{2}+\frac{j_{4}^{2}}{2}+j_{4} j_{6}+\frac{j_{5}^{2}}{2}\right], \\
& \frac{d j_{2}}{d t}=-\left[j_{2}^{2}+\frac{j_{4}^{2}}{2}-j_{4} j_{6}+\frac{j_{5}^{2}}{2}\right], \\
& \frac{d j_{3}}{d t}=-\left[j_{3}^{2}+j_{5}^{2}\right], \\
& \frac{d j_{4}}{d t}=-\left[j_{4}\left(j_{1}+j_{2}+j_{6}\right)+j_{6}\left(j_{1}-j_{2}\right)\right], \\
& \frac{d j_{5}}{d t}=-\frac{j_{5}}{2}\left[j_{1}+j_{2}+j_{3}-j_{6}\right], \\
& \frac{d j_{6}}{d t}=-j_{6}^{2} .
\end{aligned}
$$

Here $j_{i}=\rho_{0} J_{i}$, and the scaling variable is $t=\ln \bar{D}$.

Analysis of solutions of the scaling equations (20) with initial values of coupling parameters listed in Eq. (19), shows that the symmetry-breaking vertices $j_{3,5}$ are irrelevant, and the vertex $j_{2}$, which is negative at the boundary $\bar{D}=\bar{D}_{S W}$ evolves into positive domain and eventually enters the Kondo temperature. The latter is given by the following equation

$$
T_{k}=\bar{D} \exp \left\{-\frac{2}{j_{1}+j_{2}+\sqrt{2} j_{4}+2 j_{6}}\right\} .
$$

We see from this equation that both spin and pseudospin coupling contribute to the Kondo cotunneling on equal footing, so that $T_{K}$ is enhanced due to involvement of additional degrees of freedom connected with electron permutations in a triangle.

The tunnel contact reduces the symmetry of TTQD when the conditions (6) are satisfied. In this case the double orbital degeneracy of the level $E_{D E}$ is removed by tunnel coupling. Then the generic symmetry of TTQD is usual $S U(2)$ symmetry, but an accidental degeneracy between the levels $E_{D E(+)}$ and $E_{D A_{1}}$ arises under certain condition, and in this case the $S U(4)$ symmetry is restored. This situation as well as the properties of TTQD with $\mathcal{N}=2$ will be considered in more detailed forthcoming publication.

\section{CONCLUSIONS}

Dynamical symmetry is a universal tool, which allows to derive the effective spin Hamiltonians describing low-energy cotunneling with spin reversal through composite quantum dots. These dots possess both the continuous rotation symmetry in spin space and discrete rotation symmetry in real space. We considered in this paper the relatively simple example of symmetric triangular quantum dot in contact with metallic leads in a specific geometry where the dot imposes its discrete rotation symmetry on the electrons in the leads. The tunnel problem is mapped on the CoqblinSchrieffer (CS) model of magnetic impurity with "orbital" degrees of freedom. Like in the latter case, the symmetry of the Kondo center is $S U(2 n)$. The parameter $n$ characterizes additional orbital degeneracy in conventional CS model. In case of artificial triangular molecule it describes the pseudospin operator of finite clockwise/anti-clockwise rotations of TTQD in the process of electron cotunneling. 
1 L.W. Molenkamp, K. Flensberg, and M. Kemerlink, Phys. Rev. Lett. 75, 4282 (1995); F. Hofmann, T. Heinzel, D.A. Waram, J.P. Kotthaus, G. Böm, W. Klein, G. Tränkle, and G. Weimann, Phys. Rev. B 51, 13872 (1995).

2 J.J. Palacios and P. Hawrilak, Phys. Rev. B 51, 1769 (1995).

3 D. Goldhaber-Gordon, H. Shtrikman, D. Mahalu, D. Abush-Magder, U. Meirav, and M.A. Kastner, Nature 391, 156 (1998); S.M. Cronenwett, T.H. Oosterkamp, and L.P. Kouwenhoven, Science 281, 540 (1998); F. Simmel, R.H. Blick, J.P. Kotthaus, W. Wegscheider, and M. Bichler, Phys. Rev. Lett. 83, 804 (1999).

${ }^{4}$ B. Partoens and F.M. Peeters, Phys. Rev. Lett. 84, 4433 (2000); E. Anisimovas and F.M. Peeters, Phys. Rev. B 65, 233302 (2002); 66, 075311 (2002).

${ }^{5}$ K. Kikoin and Y. Avishai, Phys. Rev. Lett. 86, 2090 (2001), Ibid. Phys. Rev. B65, 115329 (2002);

6 W. Hofstetter and H Schoeller, Phys. Rev. Lett. 88, 016803 (2002)

7 T. Kuzmenko, K. Kikoin, and Y. Avishai, Phys. Rev. Lett. 89, 156602 (2002); Phys. Rev. B 69195109 (2004).

8 M. Stopa, Phys. Rev, Lett. 88, 146802 (2002)

9 A. Vidan, R.M. Westervelt, M. Stopa, M. Hanson, and A.C. Gossard, Appl. Phys. Lett. (in press)

10 D.Cox and A. Zawadovski, Adv. Phys. 47, 599 (1998)

11 B. Coqblin, J.R. Schrieffer, Phys. Rev. P185, 847 (1969).

12 F.D.M. Haldane, Phys. Rev. Lett. 40, 416 (1978).

13 P.W. Anderson, J. Phys. C 3, 2436 (1970).

${ }^{14}$ K. Kikoin, Y. Avishai and M.N. Kiselev. Dynamical symmetries in nanophysics, arXiv-cond.mat/0407063, to be published in "Progress in Nanotechnology Research", Nova Science, New York.

$15 \mathrm{Ph}$. Nozieres and A. Blandin, J. Physique 41, 193 (1980).

16 L. Borda, G. Zarand, W. Hofstetter, B.I. Halperin, and J. von Delft, Phys. Rev. Lett. 90, 026602 (2003). 